\title{
Phase II trial of docetaxel combined with nedaplatin for patients with recurrent and metastatic nasopharyngeal carcinoma
}

This article was published in the following Dove Press journal:

Drug Design, Development and Therapy

10 December 2015

Number of times this article has been viewed

\author{
Pei-Jian Peng ${ }^{1, *}$ \\ Bao-Jun Lv',* \\ Con Tang 2 ,* \\ Hai Liao ${ }^{3}$ \\ Zhong Lin' \\ Yu-Meng Liu ${ }^{4}$ \\ Zhi-Hui Wang' \\ Si-Yang Wang ${ }^{5}$ \\ Zhi-Bin Cheng ${ }^{5}$ \\ 'Department of Medical Oncology, \\ ${ }^{2}$ Department of Surgical Oncology, \\ The Fifth Affiliated Hospital of \\ Sun Yat-sen University, Zhuhai, \\ ${ }^{3}$ Department of Medical Oncology, \\ Cancer Centre, Sun Yat-sen University, \\ Guangzhou, ${ }^{4}$ Department of Oncology, \\ People's Hospital of Zhongshan City, \\ Zhongshan, ${ }^{5}$ Department of Radiation \\ Oncology, The Fifth Affiliated Hospital \\ of Sun Yat-sen University, Zhuhai, \\ Guangdong Province, People's \\ Republic of China \\ *These authors contributed equally \\ to this work
}

Purpose: This Phase II trial was designed to evaluate the efficacy and safety of docetaxel combined with nedaplatin as first-line treatment for patients with recurrent or metastatic nasopharyngeal carcinoma.

Methods: In this multicenter Phase II trial, the patients were treated with intravenous docetaxel $\left(75 \mathrm{mg} / \mathrm{m}^{2}\right.$, day 1$)$ and nedaplatin $\left(80 \mathrm{mg} / \mathrm{m}^{2}\right.$, day 1$)$, each cycle repeated every 3 weeks for two cycles at least.

Results: From January 2010 to November 2013, a total of 78 patients were recruited in this trial. Among them, 73 patients were assessable for response. The treatment was well tolerated. The main hematological adverse event was neutropenia. A total of 12 patients (15.4\%) had grade 3 or grade 4 neutropenia. Grade 3 anemia was observed in six patients $(7.7 \%)$ and no grade $3 / 4$ thrombocytopenia was observed. No Grade $3 / 4$ non-hematological toxicity was observed. There were five complete response $(6.8 \%), 43$ partial responses $(58.9 \%)$, and the overall response rate was $65.8 \%$ (95\% confidence interval [CI], 48.7\%-81.2\%). With a median follow-up period of 18.6 months, the median time to progression was 7.9 months (95\% CI, 4.2-10.8 months), median overall survival was 15.7 months (95\% CI, 11.6-18.5 months).

Conclusion: Docetaxel combined with nedaplatin offers a satisfactory clinical activity and an acceptable safety profile as first-line chemotherapy for patients with recurrent and metastatic nasopharyngeal carcinoma.

Keywords: docetaxel, nedaplatin, nasopharyngeal carcinoma

\section{Introduction}

Nasopharyngeal carcinoma (NPC) is prevalent in South China, particularly in Guangdong Province. ${ }^{1,2}$ Although many improvements in technology and equipment of radiotherapy have been achieved and concurrent chemoradiotherapy was used, the outcome of patients with locoregionally advanced NPC is still unsatisfactory. The main reason of failure is locoregional relapse and distant metastasis. ${ }^{3,4}$ The NPC is also sensitive to chemotherapy and treatment result of survival prolongation can often be achieved for recurrent and/ or metastatic disease. Cisplatin-based chemotherapy regimens are often considered as standard chemotherapy for recurrent or metastatic NPC..$^{5-7}$ However, gastrointestinal (GI) and renal toxicities are frequent with cisplatin-based regimens. Therefore, new active regimens with a favorable toxicity profile need to be explored.

Docetaxel has shown significant activity against head and neck cancer including NPC. As a single agent, docetaxel is effective in the patients with disseminated NPC who were refractory to platinum-based chemotherapy. ${ }^{8}$ In addition, a clinical study has indicated that docetaxel plus cisplatin is active as first-line chemotherapy in the patients with metastatic NPC. ${ }^{9}$ Nedaplatin is a second-generation platinum derivative, 
which has similar antitumor activity to cisplatin with less nephrotoxicity and GI toxicity. ${ }^{10-12}$ Also, our recent Phase II study of nedaplatin plus capecitabine has shown satisfactory antitumor activity as a salvage chemotherapy for cisplatinrefractory recurrent and metastatic NPC. ${ }^{13}$

Based on these promising results, we designed a multicenter, open-label, single-arm Phase II trial to evaluate the efficacy and toxicity of docetaxel plus nedaplatin as first-line chemotherapy for the patients with recurrent or metastatic NPC.

\section{Patients and methods}

\section{Patients' eligibility}

Eligibility criteria included histologically confirmed recurrent and/or metastatic NPC, age from 18 to 75 years, Eastern Cooperative Oncology Group (ECOG) performance status of zero to two, a life expectancy $\geqq 12$ weeks, and at least one measurable lesion according to the RECIST (response evaluation criteria in solid tumor) version 1.0 (nasopharyngeal tumor was considered as measurable disease, and the metastatic bone diseases were considered as unmeasurable lesions). Earlier treatment of chemotherapy for advanced disease was not permitted. However, adjuvant, neoadjuvant, or concurrent treatments were permitted, provided that the chemotherapy was completed at least 6 months earlier at the start of this trial. Adequate liver (bilirubin level $\leqq 1.5 \mathrm{mg} / \mathrm{dL}$, aspartate aminotransferase or alanine aminotransferase levels $\leqq 2.5$ times the upper limit of normal), renal (serum creatinine level $\leqq 1.5 \mathrm{mg} / \mathrm{dL}$ ), and bone marrow function (hemoglobin level $\geqq 10 \mathrm{~g} / \mathrm{dL}$, white blood cell count $\geqq 4,000 / \mu \mathrm{L}$, neutrophil count $\geqq 1,500 / \mu \mathrm{L}$, and platelet count $\geqq 100,000 / \mu \mathrm{L}$ ) were required. Written, informed consent was obtained before enrollment, and the study was approved by the local ethical committees from the Fifth Affiliated Hospital of Sun-Yat-Sen University, Cancer Center of Sun-Yat-Sen University, and the People's Hospital of Zhongshan City.

\section{Treatment}

Docetaxel at a dose of $75 \mathrm{mg} / \mathrm{m}^{2}$ was infused during a 1-hour on day 1 . Based on the recently published Phase I/II studies, nedaplatin was administered intravenously during a 2-hour infusion on day 1 (after the docetaxel infusion) at a dose of $80 \mathrm{mg} / \mathrm{m}^{2} .^{12-14}$ Prior to docetaxel administration, a serotonin antagonist was routinely used to prevent emesis. This regimen was repeated every 3 weeks. In responders, chemotherapy was repeated for a maximum of six cycles. The treatment was discontinued when there were unacceptable adverse reactions, evidence of disease progression at any time, patients' voluntary withdrawal, or after six cycles of chemotherapy.

\section{Evaluation of efficacy}

The primary objective was response rate (RR), and secondary objectives were toxicity, time to progression (TTP), and overall survival (OS). Tumor response was evaluated every two cycles during the chemotherapy and then every 3 months after the completion of the chemotherapy using RECIST.

\section{Evaluation of safety and dose modification}

Toxicities were assessed using National Cancer Institute Common Toxicity Criteria (version 3.0) before each treatment cycle. Complete physical examination, serum chemistry analysis, chest X-ray, and a computed tomography scan of target sites were performed before the start of this study. Before each treatment cycle, the patients were required to have a neutrophil count $\geqq 1,500 / \mu \mathrm{L}$, a platelet count $\geqq 100,000 / \mu \mathrm{L}$, adequate hepatic function, and renal function. If the chemotherapy was interrupted by more than 3 weeks, the patients were taken off from the study.

In case of toxicities with grade 3 intensity or higher, the dose was modified according to the following criteria: the dose of docetaxel and nedaplatin for the subsequent cycle was reduced by $20 \%$ in the case of a repeated any grade 3 toxicity, and reduced by $40 \%$ in the case of a repeated any grade 4 toxicity. If a dose reduction exceeding $40 \%$ was required, the patients were excluded from the study. The use of growth factor was permitted.

\section{Statistical analysis}

This trial was proposed according to Simon's ${ }^{15}$ two-stage Phase II optimal trial design. Based on a hypothesis that a RR of $40 \%$ was expected to detect when compared with a minimal, clinically meaningful RR of $20 \%$, the total sample size of 48 patients was necessary. All recruited patients were included in the intention-to-treat analysis of efficacy and toxicity. The overall RR was calculated with $95 \%$ confidence intervals (CIs). The TTP and OS were estimated by the Kaplan-Meier method. The TTP was measured from the date of entry into the study until the date of progression, and the OS was calculated from the date of entry to the date of the last follow-up or death. The statistical analyses were performed using an SPSS software package (SPSS 16.0, SPSS Inc., Chicago, IL, USA).

\section{Results}

\section{Patient characteristics}

From January 2010 to November 2013, 78 patients were recruited in this trial from The Fifth Affiliated Hospital of Sun Yat-sen University, Cancer Center of Sun Yat-sen 
Table I Patients characteristics

\begin{tabular}{|c|c|c|}
\hline Characteristics & $\begin{array}{l}\text { Number of patients } \\
(n=78)\end{array}$ & $\%$ \\
\hline Age (years) & Median 47 (range, 3I-68) & \\
\hline \multicolumn{3}{|l|}{ Sex } \\
\hline Female & 29 & 37.2 \\
\hline Male & 49 & 62.8 \\
\hline \multicolumn{3}{|l|}{ ECOG performance status } \\
\hline 0 & 21 & 26.9 \\
\hline I & 45 & 57.7 \\
\hline 2 & 12 & 15.4 \\
\hline \multicolumn{3}{|l|}{ WHO histology type } \\
\hline Type III & 78 & 100 \\
\hline \multicolumn{3}{|l|}{ Tumor-involved site } \\
\hline Nasopharyngeal & 4 & 5.1 \\
\hline Lymph node & 10 & 12.8 \\
\hline Lungs & 42 & 53.8 \\
\hline Liver & 31 & 39.7 \\
\hline Bone & 9 & 11.5 \\
\hline \multicolumn{3}{|l|}{ Relapse pattern } \\
\hline Recurrent & 6 & 7.7 \\
\hline Metastatic & 64 & 82.0 \\
\hline Recurrent and metastatic & 8 & 10.3 \\
\hline \multicolumn{3}{|l|}{ Number of involved site } \\
\hline 1 & 53 & 67.9 \\
\hline 2 & 19 & 24.4 \\
\hline 3 & 6 & 7.7 \\
\hline \multicolumn{3}{|l|}{ Initial received treatment (cases) } \\
\hline Treatment-naive & 12 & 15.4 \\
\hline Radiation & 66 & 84.6 \\
\hline $\begin{array}{l}\text { Induction/concurrent/adjuvant } \\
\text { chemotherapy }\end{array}$ & 38 & 48.7 \\
\hline
\end{tabular}

Abbreviations: ECOG, Eastern Cooperative Oncology Group; WHO, World Health Organization.

University, and the People's Hospital of Zhongshan City. The characteristics of the patients are summarized in Table 1.

\section{Response and survival}

Among 78 patients, only 73 (93.6\%) were assessable for response. Three patients were lost to follow-up after the second cycle of the treatment, and two patients withdrew their consents because of toxicities after one cycle. All efficacy data are reported using the intention-to-treat principle. Among the treated patients, 5 patients $(6.8 \%)$ had complete response (CR), 43 patients $(58.9 \%)$ had partial responses (PR), 22 patients (30.1\%) had stable disease, and three patients $(4.1 \%)$ had progressive disease. The overall RR (CR + PR) was $65.8 \%$ (95\% CI, 48.7\%-81.2\%). With a median follow-up time of 18.6 months (range 2.5-43 months), median TTP was 7.9 months (95\% CI, 4.2-10.8 months; Figure 1), median OS for all the patients was 15.7 months (95\% CI, 11.6-18.5 months; Figure 2), with a 1-year survival rate of $69 \%$ and 2 -year survival rate

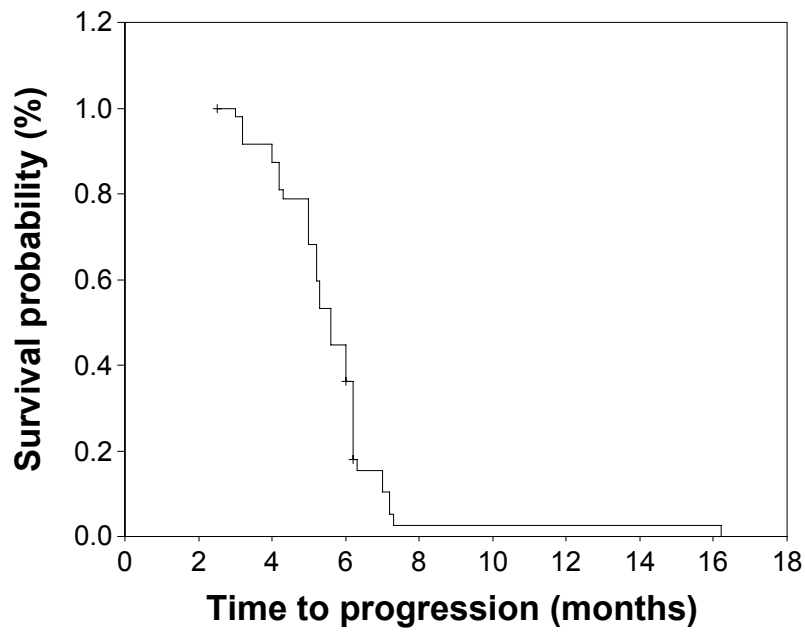

Figure I Kaplan-Meier curve of progression-free survival.

of $33 \%$. Three patients with multiple metastases, who were treated with docetaxel plus nedaplatin regimen, survived more than 38 months and are still alive.

\section{Toxicity}

A total of 318 cycles of docetaxel plus nedaplatin were delivered to the 78 patients who were assessable for safety, with the median number of four cycles (range, 1-6 cycles) administered per patient. Dose reduction was needed in ten cycles (of 318) and cycle's delay was needed in 14 cycles (of 318). The toxicity frequencies are listed in Table 2. The main hematological toxicity was neutropenia with 12 patients $(15.4 \%)$ experiencing grade $3 / 4$ neutropenia. Only three patients $(3.8 \%)$ developed febrile neutropenia. Six patients $(7.7 \%)$ experienced grade 3 anemia and no grade $3 / 4$ thrombocytopenia were observed. The main

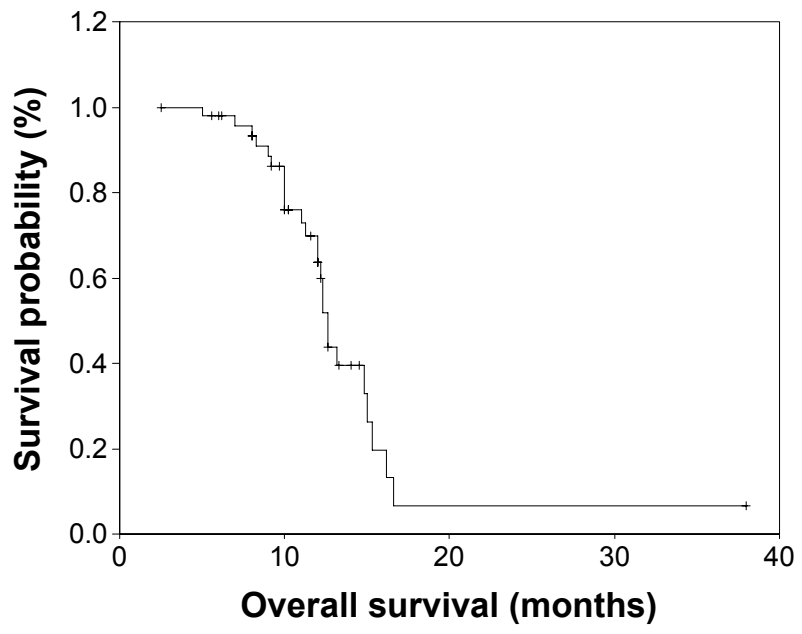

Figure 2 Kaplan-Meier curve of overall survival. 
Table 2 Treatment-related adverse events $(n=78)$

\begin{tabular}{lllll}
\hline Adverse events & \multicolumn{4}{c}{ NCI-CTC grade (\% of patients) } \\
\cline { 2 - 5 } & Grade I & Grade 2 & Grade 3 & Grade 4 \\
\hline Hematological & $36(46.2)$ & $30(38.5)$ & $9(11.5)$ & $3(3.8)$ \\
$\quad$ Leucopenia & $27(34.6)$ & $18(23.1)$ & $6(7.7)$ & $0(0.0)$ \\
$\quad$ Anemia & $29(37.2)$ & $12(15.4)$ & $0(0.0)$ & $0(0.0)$ \\
$\quad \begin{array}{llll}\text { Thrombocytopenia } \\
\text { Non-hematological }\end{array}$ & & & & \\
$\quad$ Nausea & $41(52.5)$ & $23(29.5)$ & $0(0.0)$ & $0(0.0)$ \\
Vomiting & $38(48.7)$ & $17(21.8)$ & $0(0.0)$ & $0(0.0)$ \\
Fatigue & $22(28.2)$ & $14(17.9)$ & $0(0.0)$ & $0(0.0)$ \\
$\quad$ Neuropathy & $13(16.7)$ & $8(10.3)$ & $0(0.0)$ & $0(0.0)$ \\
$\quad$ Neutropenia fever & $0(0.0)$ & $3(3.8)$ & $0(0.0)$ & $0(0.0)$ \\
\hline
\end{tabular}

Abbreviation: $\mathrm{NCl}-\mathrm{CTC}$, National Cancer Institute Common Toxicity Criteria.

non-hematological toxicities were nausea ( $82 \%)$ and vomiting $(70.5 \%)$. No grade $3 / 4$ nausea and vomiting were observed. Hepatic, renal, neuropathy, fatigue, and other toxicities were mild.

\section{Discussion}

Chemotherapy has played an important palliative treatment role for recurrent and/or metastatic NPC. ${ }^{3,4,16}$ However, no standard chemotherapy regimen for advanced NPC has been established. A lot of chemotherapy regimens have been investigated in an attempt to prolong survival and improve the quality of life. The cisplatin-containing regimens are commonly considered the "gold standard". The doublet combination of cisplatin with 5-FU, capecitabine, taxane, or gemcitabine were commonly employed as first-line chemotherapy regimen. ${ }^{7,16-22}$ Despite the well-documented sensitivity of NPC to a variety of cisplatin-containing regimens, treatment with cisplatin can induce severe toxicity including severe GI toxicity, neurologic toxicity, and myelosuppression.

Docetaxel is active against several solid tumors including head and neck cancers. Its activity in NPC has been investigated as a single drug or in combination with platinum in both the metastatic/recurrent and locoregionally advanced settings ${ }^{8,9,23}$ Ngeow et al ${ }^{8}$ confirmed the single-agent activity of docetaxel in the setting of heavily pretreated metastatic NPC. The median progression free survival of 5.8 months and OS of 12.8 months are encouraging. Nedaplatin is a secondgeneration platinum complex with lower renal and GI toxicities than cisplatin. In contrast to cisplatin, nedaplatin does not require a large amount of fluid infusion. Moreover, it was reported that nedaplatin is at least as effective as cisplatin for head and neck cancer. ${ }^{10-12,24}$ So, a combination of docetaxel and nedaplatin to treat NPC seemed to be very rational.

In our current study, this regimen showed a RR of $65.8 \%$ with the median TTP of 7.9 months and median OS of
15.7 months, and 1-year survival rate were $69 \%$. In contrast to other clinical trials, the combination of docetaxel and nedaplatin seems to be an effective regimen as first-line chemotherapy for patients with recurrent and metastatic NPC. ${ }^{9,17-23}$ Chua et $\mathrm{al}^{9}$ reported a Phase II study in which the patients with metastatic NPC received docetaxel and cisplatin in a 3-week cycle. The overall RR was $62.5 \%$ with a median TTP of 5.6 months and median OS of 12.4 months. Ngan et a ${ }^{18}$ reported a Phase II trial using gemcitabine plus cisplatin for the treatment of recurrent or metastatic NPC. There were nine CRs and 23 PRs in 44 patients, achieving an overall RR of $73 \%$. With a median follow-up of 17.2 months, $62 \%$ survived 1 year, while $36 \%$ were alive and progression free. In a study treating patients with metastatic NPC with capecitabine and cisplatin, Li et a ${ }^{19}$ reported similar survival data (an overall RR of $36 \%$, a median progression free survival of 5.6 months, and a median OS of 11.9 months). From our current study results, whether docetaxel in combination with nedaplatin is indeed comparable or superior to other regimens as a first-line treatment in terms of efficacy has not yet been fully demonstrated and requires further prospective randomized study.

Compared with cisplatin-based regimens, docetaxel plus nedaplatin regimen showed lower GI toxicities and did not require a large amount of fluid infusion. ${ }^{17-19,23}$ The main hematological toxicity was neutropenia with 12 patients $(15.4 \%)$ experiencing grade $3 / 4$ neutropenia. Only three patients (3.8\%) developed febrile neutropenia. Six patients (7.7\%) experienced grade 3 anemia and no grade 3/4 thrombocytopenia were observed. The main non-hematological toxicities were nausea ( $82 \%$ ) and vomiting $(70.5 \%)$. No grade $3 / 4$ nausea and vomiting were observed. Hepatic, renal, neuropathy, fatigue, and other toxicities were mild. These toxicities were well tolerated. Only two patients were discontinued from the study due to toxic effects. No treatment-related death occurred during this study. However, in a Phase II study treating patients with metastatic NPC with docetaxel and cisplatin reported by Chua et al, ${ }^{9}$ treatment-related adverse events seemed to be severe, hematological toxicity with grade 4 neutropenia occurred in $78.9 \%$ patients.

\section{Conclusion}

In summary, our results indicate that the combination of docetaxel and nedaplatin is a useful and well-tolerated treatment as first-line chemotherapy for recurrent and metastatic NPC. Because of the relatively mild toxicity, this combination chemotherapy seemed to be much more patient-friendly comparing with other cisplatin-containing regimens. However, as data with a Phase II study is limited, 
further prospective randomized trials are needed to assess the efficacy of docetaxel and nedaplatin to compare it with other cisplatin-based chemotherapy regimens in recurrent and metastatic NPC.

\section{Disclosure}

The authors report no conflicts of interest in this work.

\section{References}

1. Chang ET, Adami HO. The enigmatic epidemiology of nasopharyngeal carcinoma. Cancer Epidemiol Biomarkers Prey. 2006;15(10): 1765-1777.

2. Cao SM, Simons MJ, Qian CN. The prevalence and prevention of nasopharyngeal carcinoma in China. Chin J Cancer. 2011;30: $114-119$

3. Zhang L, Chen QY, Liu H, Tang LQ, Mai HQ. Emerging treatment options for nasopharyngeal carcinoma. Drug Des Devel Ther. 2013; 7 : $37-52$.

4. Lee AW, Ng WT, Chan YH, Sze H, Chan C, Lam TH. The battle against nasopharyngeal cancer. Radiother Oncol. 2012;104(3):272-278.

5. Ma BB, Chan AT. Recent perspectives in the role of chemotherapy in the management of advanced nasopharyngeal carcinoma. Cancer. 2005;103(1):22-31.

6. Lee AW, Sze WM, Au JS, et al. Treatment results for nasopharyngeal carcinoma in the modern era: the Hong Kong experience. Int J Radiat Oncol Biol Phys. 2005;61(4):1107-1116.

7. Suárez C, Rodrigo JP, Rinaldo A, Langendijk JA, Shaha AR, Ferlito A. Current treatment options for recurrent nasopharyngeal cancer. Eur Arch Otorhinolaryngol. 2010;267(12):1811-1824.

8. Ngeow J, Lim WT, Leong SS, et al. Docetaxel is effective in heavily pretreated patients with disseminated nasopharyngeal carcinoma. Ann Oncol. 2011;22(3):718-722.

9. Chua DT, Sham JS, Au GK. A phase II study of docetaxel and cisplatin as first-line chemotherapy in patients with metastatic nasopharyngeal carcinoma. Oral Oncol. 2005;41(6):589-595.

10. Sasaki Y, Shinkai T, Eguchi K, et al. Prediction of the antitumor activity of new platinum analogs based on their ex vivo pharmacodynamics as determined by bioassay. Cancer Chemother Pharmacol. 1991;27(4): 263-270.

11. Kawai Y, Taniuchi S, Okahara S, Nakamura M, Gemba M. Relationship between cisplatin or nedaplatin-induced nephrotoxicity and renal accumulation. Biol Pharm Bull. 2005;28(8):1385-1388.
12. Alberts DS, Fanta PT, Running KL, et al. In vitro phase II comparison of the cytotoxicity of a novel platinum analog, nedaplatin (254-S), with that of cisplatin and carboplatin against fresh, human ovarian cancers. Cancer Chemother Pharmacol. 1997;39(6):493-497.

13. Peng PJ, Ou XQ, Chen ZB, et al. Multicenter phase II study of capecitabine combined with nedaplatin for recurrent and metastatic nasopharyngeal carcinoma patients after failure of cisplatin-based chemotherapy. Cancer Chemother Pharmacol. 2013;72(2):323-328.

14. Kurita H, Yamamoto E, Nozaki S, Wada S, Furuta I, Kurashina K. Multicenter phase I trial of induction chemotherapy with docetaxel and nedaplatin for oral squamous cell carcinoma. Oral Oncol. 2004; 40(10):1000-1006.

15. Simon R. Optimal two-stage designs for phase II clinical trials. Control Clin Trials. 1989;10(1):1-10.

16. Ma BB, Hui EP, Chan AT. Systemic approach to improving treatment outcome in nasopharyngeal carcinoma: current and future directions. Cancer Sci. 2008;99(7):1311-1318.

17. Au E, Ang PT. A phase II trial of 5-fluorouracil and cisplatinum in recurrent or metastatic nasopharyngeal carcinoma. Ann Oncol. 1994; 5(1):87-89.

18. Ngan RK, Yiu HH, Lau WH, et al. Combination gemcitabine and cisplatin chemotherapy for metastatic or recurrent nasopharyngeal carcinoma: report of a phase II study. Ann Oncol. 2002;13(8):1252-1258.

19. Li YH, Wang FH, Jiang WQ, et al. Phase II study of capecitabine and cisplatin combination as first-line chemotherapy in Chinese patients with metastatic nasopharyngeal carcinoma. Cancer Chemother Pharmacol. 2008;62(3):539-544.

20. Yeo W, Leung TWT, Chan ATC, et al. A phase II study of combination paclitaxel and carboplatin in advanced nasopharyngeal carcinoma. Eur J Cancer. 1998;34(13):2027-2031.

21. Zhang L, Zhang Y, Huang PY, Xu F, Peng PJ, Guan ZZ. Phase II clinical study of gemcitabine in the treatment of patients with advanced nasopharyngeal carcinoma after the failure of platinum-based chemotherapy. Cancer Chemother Pharmacol. 2008;61(1):33-38.

22. Wang CC, Chang JY, Liu TW, Lin CY, Yu YC, Hong RL. Phase II study of gemcitabine plus vinorelbine in the treatment of cisplatin-resistant nasopharyngeal carcinoma. Head Neck. 2006;28(1):74-80.

23. Hui EP, Ma BB, Leung SF, et al. Randomized phase II trial of concurrent cisplatin-radiotherapy with or without neoadjuvant docetaxel and cisplatin in advanced nasopharyngeal carcinoma. J Clin Oncol. 2009;27(2):242-249.

24. Sasaki Y, Tamura T, Eguchi K, et al. Pharmacokinetics of (glycolate$0,0^{\prime}$ )-diammine platinum (II), a new platinum derivative, in comparison with cisplatin and carboplatin. Cancer Chemother Pharmacol. 1989;23(4):243-246.

\section{Publish your work in this journal}

Drug Design, Development and Therapy is an international, peerreviewed open-access journal that spans the spectrum of drug design and development through to clinical applications. Clinical outcomes, patient safety, and programs for the development and effective, safe, and sustained use of medicines are a feature of the journal, which

\section{Dovepress}

has also been accepted for indexing on PubMed Central. The manuscript management system is completely online and includes a very quick and fair peer-review system, which is all easy to use. Visit http://www.dovepress.com/testimonials.php to read real quotes from published authors. 